\title{
1 GABAergic signaling in human and murine NK cells upon challenge with \\ 2 Toxoplasma gondii
}

4 Amol K. Bhandage ${ }^{1}$, Laura M. Friedrich ${ }^{1,2}$, Sachie Kanatani ${ }^{1}$, Simon Jakobsson-Björkén ${ }^{2}$, J.

5 Ignacio Escrig-Larena ${ }^{2}$, Arnika K. Wagner ${ }^{3}$, Benedict J. Chambers ${ }^{2}$, Antonio Barragan ${ }^{1}$

$7{ }^{1}$ Department of Molecular Biosciences, The Wenner-Gren Institute, Stockholm University,

8 Stockholm, Sweden

$9 \quad{ }^{2}$ Center for Infectious Medicine, Department of Medicine, Karolinska Institutet, Karolinska

10 University Hospital, Huddinge, Stockholm, Sweden

$11{ }^{3}$ Center for Hematology and Regenerative Medicine, Department of Medicine Huddinge,

12 Karolinska Institutet, Karolinska University Hospital, Huddinge, Stockholm, Sweden

14 Summary sentence: In response to infection challenge, NK cells synthesize and

15 secrete $\gamma$-aminobutyric acid (GABA), which impacts NK cell and dendritic cell functions via 16 GABA-A receptors.

18 Running title: GABAergic signaling in NK cells

20 Corresponding author: Antonio Barragan, Dept. of Molecular Biosciences (MBW), The Wenner-

21 Gren Institute, Stockholm University, Svante Arrhenius väg 20C, SE-106 91 Stockholm, Sweden

22 Tel: +46-8-164156. E-mail: antonio.barragan@su.se

24 Keywords: Lymphocyte, Apicomplexa, GABA-A receptor, immunomodulation, cell migration, 25 neurotransmitter, Host-pathogen 


\section{Abstract}

27 Protective cytotoxic and proinflammatory cytokine responses by natural killer (NK) cells impact

28 the outcome of infections by Toxoplasma gondii, a common parasite in humans and other

29 vertebrates. However, T. gondii can also sequester within NK cells and downmodulate their

30 effector functions. Recently, the implication of $\gamma$-aminobutyric acid (GABA) signaling in

31 infection and inflammation-related responses of mononuclear phagocytes and $T$ cells has

32 become evident. Yet, the role of GABAergic signaling in NK cells has remained unknown. Here,

33 we report that human and murine NK cells synthesize and secrete GABA in response to

34 infection challenge. Parasitized NK cells secreted GABA while activation stimuli, such as IL-12/IL-

3518 or parasite lysates, failed to induce GABA secretion. GABA secretion by NK cells was

36 associated to a transcriptional upregulation of GABA synthesis enzymes (GAD65/67) and was

37 abrogated by GAD-inhibition. Further, NK cells expressed GABA-A receptor subunits and GABA

38 signaling regulators, with transcriptional modulations taking place upon challenge with $T$.

39 gondii. Exogenous GABA and GABA-containing supernatants from parasitized dendritic cells

40 (DCs) impacted NK cell function by reducing the degranulation and cytotoxicity of NK cells.

41 Conversely, GABA-containing supernatants from NK cells enhanced the migratory responses of

42 parasitized DCs. This enhanced DC migration was abolished by GABA-A receptor antagonism or

43 GAD-inhibition and was reconstituted by exogenous GABA. Jointly, the data show that NK cells

44 are GABAergic cells and that GABA hampers NK cell cytotoxicity in vitro. We hypothesize that

45 GABA secreted by parasitized immune cells modulates the immune responses to $T$. gondii 46 infection. 


\section{Introduction}

Г-aminobutyric acid (GABA), the main inhibitory neurotransmitter of the vertebrate brain, has also been attributed motogenic functions outside the central nervous system, including immune cell migration in infection and metastasis of cancer cells ${ }^{1-4}$. GABA-A receptors (GABA-A R) are ionotropic chloride $\left(\mathrm{Cl}^{-}\right)$channels composed from pentameric combinations of 19 different subunits ${ }^{5}$ and whose GABA signaling functions are regulated by cation-chloride co-transporters (CCCS) ${ }^{6}$. GABAergic cells, for example neurons, synthesize GABA via glutamate decarboxylases (GAD65/67) and metabolize GABA by GABA-transaminase (GABA-T) ${ }^{7}$. Further, GABA transporters (GAT) shuttle GABA in and out of cells ${ }^{8}$.

The roles of GABAergic signaling have been extensively studied in neurons and astrocytes ${ }^{9,10}$ but remain chiefly unexplored in cells of the immune system ${ }^{11}$. Recent reports have elucidated that immune cells, such as T cells, macrophages, monocytes and dendritic cells (DCs), express GABAergic signaling components and are responsive to GABA, which has been attributed immunomodulatory functions ${ }^{2,3,12,13}$. However, GABAergic signaling in natural killer (NK) cells has remained elusive.

Toxoplasma gondii is an obligate intracellular protozoan that infects warm-blooded vertebrates, including humans and rodents ${ }^{14}$. Chronic carriage of $T$. gondii without major or no symptomatology is common. However, systemic dissemination of $T$. gondii can cause lifethreatening encephalitis in immune-compromised patients, disabling disease in the developing foetus, and ocular manifestations ${ }^{15,16}$. The tachyzoite parasite stage actively invades and replicates within nucleated cells in the host ${ }^{17}$, including immune cells such as dendritic cells (DCS), T cells or NK cells ${ }^{18-20}$. Upon infection of DCs and other mononuclear phagocytes, $T$. gondii promotes migratory activation of parasitized phagocytes through GABAergic signaling to facilitate parasite dissemination via a Trojan horse mechanism ${ }^{3,18,21}$.

NK cells play important roles in $T$. gondii infection, with an impact on innate, adaptive and regulative responses ${ }^{22}$. Early studies showed that NK cells, alike DCs, have a protective role in $T$. gondii infection as they serve as an early source of Interleukin-12 (IL-12) and Interferon- $\gamma$ $(\text { IFN- } \gamma)^{23-25}$. IFN- $\gamma$ responses lead to the activation and differentiation of macrophages and DCs which enhances the killing of the parasite and supports the activation of the T cell responses 26,27 . In this setting, NK cells home to lymphoid organs where they interact with phagocytes ${ }^{28}$, display enhanced motility ${ }^{29}$ and are able to kill $T$. gondii-infected target cells in vitro and in vivo 
78 20. Interestingly, NK cell-mediated killing of parasitized DCs led to rapid egress of viable

79 parasites which in turn infected the effector NK cells ${ }^{20}$. Conversely, parasitized NK cells

80 exhibited impaired recognition of target cells and reduced cytokine release ${ }^{30}$.

81 Yet, the host-pathogen interplay between T. gondii and parasitized NK cells and DCs

82 remains elusive. Here, we report that human and murine NK cells express the components of a

83 GABAergic machinery and secrete GABA upon challenge with $T$. gondii tachyzoites. We test the

84 functional implications of GABA secretion on DC migration and effector functions of NK cells.

\section{Methods}

\section{$87 \quad 2.1$ Ethics statement}

88 The Regional Ethics Committee, Stockholm, Sweden, approved protocols involving human cells.

89 All donors received written and oral information upon donation of blood at the Karolinska

90 University Hospital. All the animal experimentation procedures involving infection and

91 extraction of cells/organs from mice were approved by Regional Animal Research Ethical Board,

92 Stockholm, Sweden in concordance with in EU legislation (permit numbers 9707/2018 and

$9314458 / 2019)$.

\subsection{Experimental animals}

96 Six to 10-week-old C57BL/6NCrl mice were purchased from Charles River (Sulzfeld, Germany)

97 and maintained or bred under pathogen-free conditions at Experimental Core Facility (ECF),

98 Stockholm University, Sweden. Six to 10 -week-old B6.RAG $1^{-1-}$ mice ${ }^{31}$ and $\mathrm{C} 57 \mathrm{BL6} / \mathrm{J}$ mice

99 (Janvier Laboratories, France) were maintained at Astrid Fagraeus Laboratories, Karolinska

100 Institutet.

101

\section{$102 \quad 2.3$ Parasites and cell lines}

103 T. gondii GFP-expressing RH-LDM (type I) and ME49/PTG (type II) ${ }^{32,33}$ were maintained by serial

104 2-day passaging in human foreskin fibroblast (HFF-1, ATCC ${ }^{\circledR}$ SCRC-1041 ${ }^{\mathrm{TM}}$, American Tissue

105 Culture Collection) monolayers cultured in DMEM (Thermofisher scientific) with $10 \%$ fetal

106 bovine serum (FBS; Sigma-Aldrich), gentamicin (20 $\mathrm{g} / \mathrm{ml}$; Gibco), glutamine (2 mM; Gibco), and

107 HEPES (0.01 M; Gibco). NK-92 cells (ATCC ${ }^{\circledR}$ CRL-2407 ${ }^{\mathrm{TM}}$ ) were grown in stem cell growth

108 medium (CellGro; CellGenix, Freiburg, Germany) with 20\% heat-inactivated FBS (Gibco, Life 
109 Technologies, Carlsbad, CA, USA) and $1000 \mathrm{U} / \mathrm{mL}$ of Proleukin (Novartis, Basel, Switzerland) or

110 IL-2 (Miltenyi, Bergisch Gladbach, Germany). YAC-1 cells (ATCC ${ }^{\circledR}$ TIB-160 ${ }^{\text {TM}}$ ) and K-562 cells

111 (ATCC ${ }^{\circledR}$ CCL-243 $^{\text {TM }}$ ) were maintained in RPMI 1640; 10 mM HEPES, $2 \times 10^{-5}$ M 2-mercapto-

112 ethanol (2-ME), 10\% FBS, $100 \mathrm{U} / \mathrm{ml}$ penicillin, $100 \mathrm{U} / \mathrm{ml}$ streptomycin with 500U/ml IL-2.

113

\section{$114 \quad 2.4$ Primary cells}

115 NK cells. Single-cell suspensions from spleens were depleted of erythrocytes, and NK cells were 116 positively sorted using anti-DX5 ${ }^{+}$magnetic beads or by negative sorting using MACS separation,

117 according to the manufacturer's instructions (Miltenyi Biotec, Bergisch Gladbach, Germany).

118 Cells were resuspended in RPMI 1640; $10 \mathrm{mM}$ HEPES, $2 \times 10^{-5} \mathrm{M} 2-\mathrm{ME}, 10 \% \mathrm{FBS}, 100 \mathrm{U} / \mathrm{ml}$ 119 penicillin, $100 \mathrm{U} / \mathrm{ml}$ streptomycin with $1000 \mathrm{U} / \mathrm{ml}$ mouse IL-2 (Immunotools). For mRNA 120 expression by real-time quantitative PCR, NK cells were first isolated by negative sorting using 121 NK cell isolation kit (Miltenyi Biotech) and further purified by flow cell sorting following 122 labelling with anti-CD3 and anti-NK1.1 (FACS Aria Fusion, BD Biosciences). Cell isolations with 123 purity ranging between 96,1-99,8\% were used (Supplementary Fig. 1). Human NK cells were 124 isolated from peripheral blood mononuclear cells using human NK cells isolation kit (Miltenyi 125 Biotech). Cells were then incubated overnight in $100 \mathrm{U} / \mathrm{ml} \mathrm{IL-2} \mathrm{(Peprotech)} \mathrm{overnight} \mathrm{prior} \mathrm{to}$ 126 being used in experiments. Human NK cells challenged with GFP-expressing T. gondii (RH-LDM) 127 were sorted (FACS Aria Fusion, BD Biosciences) based on GFP-expression and CD56 expression. $128 \mathrm{GFP}^{+}$cells (defined as infected) and GFP- cells (defined as bystander) were collected and 129 incubated over night before supernatants were collected and analyzed by GABA-ELISA.

130 DCs. Mouse bone marrow-derived DCs (mBMDCs) were generated as previously described ${ }^{3}$. 131 Briefly, bone marrow cells extracted from legs of 6-10-week-old C57BL/6 mice (Charles River) 132 were cultivated in RPMI 1640 with 10\% FBS, gentamicin, glutamine and HEPES, additionally 133 supplemented with recombinant mouse GM-CSF (10 ng/ml; Peprotech) at $37^{\circ} \mathrm{C}$. Medium was 134 replenished on days 2 and 4. Loosely adherent cells harvested on day 6-8 were used for 135 experiments.

1372.5 Real-time quantitative PCR

138 Total RNAs were extracted using Direct-zol miniprep RNA kits (Zymo Research) with TRI reagent 139 (Sigma-Aldrich) and first-strand cDNA was synthesized using Superscript IV (Invitrogen) using a 140 standard protocol. Real-time quantitative PCR (qPCR) was performed in QuantStudio 5384 
141 Optical well plate system (Applied Biosystem) in a standard 10 ul with the 2X SYBR FAST qPCR

142 Master Mix (Sigma-Aldrich) with gene specific primers (Supplementary Table 1) with a standard

143 amplification and melt curve protocol. When indicated, primers were validated on mouse

144 whole brain homogenates, prepared as previously described. ${ }^{34}$. Relative expression $\left(2^{-\Delta c t}\right)$ was

145 determined for each target in relation to a normalization factor, geometric mean of reference

146 genes, TATA-binding protein (TBP) and importin 8 (IPO8). Heat maps represent transcriptional

147 changes in mRNA expression $\left(2^{-\Delta c t}\right)$ upon challenge with $T$. gondii tachyzoites (RH-LDM).

\subsection{GABA enzyme-linked immunosorbent assays}

150 NK cells were plated at a density of $1 \times 10^{6}$ cells $/ \mathrm{ml}$ and challenged with freshly-egressed $T$. 151 gondii tachyzoites (RH-LDM, MOI 2, $24 \mathrm{~h}$ ) in presence or absence of GABA synthesis inhibitor 152 semicarbazide (SC, $50 \mu \mathrm{M}$, Sigma-Aldrich). Cells were also challenged for $24 \mathrm{~h}$ with $\mathrm{MOI}-$ 153 equivalent numbers of heat-inactivated $\left(\mathrm{HI}\right.$, at $56^{\circ} \mathrm{C}$ for $\left.30 \mathrm{~min}\right)$ tachyzoites or sonicated 154 tachyzoites (30 s at 10 microns; Soniprep 150, MSE), supernatants released by tachyzoites in $1 \mathrm{~h}$ 155 or, in presence of recombinant mouse IL-12 (100 ng/ml, Peprotech, Rocky Hill, NJ) and 156 recombinant mouse IL-18 (100 ng/ml, Biosource, Brussels, Belgium). GABA concentrations in 157 supernatants were quantified from the standard curve generated at a wavelength of $450 \mathrm{~nm}$ 158 (VMax ${ }^{\circledast}$ Kinetic ELISA Microplate Reader, Molecular Devices) by ELISA (Labor Diagnostica Nord, 159 Nordhorn, Germany) as described previously ${ }^{3}$.

\subsection{Motility assays}

162 Cell motility analyses was performed as previously described ${ }^{3,35}$. Briefly, mouse bone marrow163 derived DCs were challenged with freshly-egressed ME49/PTG (MOI 3, 4-6 h) tachyzoites. 164 Combined treatments were performed, as indicated in figure legend, with GABA synthesis 165 inhibitor $(\mathrm{SC}, 50 \mu \mathrm{M})$, supernatants from $T$. gondii-challenged non-treated or SC-treated mNK 166 cells (added at medium:supernatant ratio of 1:1), GABA-A R inhibitor picrotoxin (50 $\mu \mathrm{M}$, Sigma167 Aldrich) or GABA (5 $\mu \mathrm{M}$, Sigma-Aldrich). Cells were then imaged in 96-well plates every min for 168 60-90 min (Zeiss Observer Z.1). Motility tracks for 50-60 cells per treatment were analyzed for 169 each experiment by manual tracking in ImageJ software. Motility plots were generated with 170 chemotaxis and migration stand-alone tool (version 2.0, IBIDI). X-and y-axes in the plots show 171 distances in $\mu \mathrm{m}$. The Box-and-whisker dot plots represent median velocities $(\mu \mathrm{m} / \mathrm{min})$ with 
172 boxes marking $25^{\text {th }}$ to $75^{\text {th }}$ percentile and whiskers marking $10^{\text {th }}$ and $90^{\text {th }}$ percentiles of the

173 datasets. Grey circles represent velocities from individual cells.

\section{$175 \quad 2.8$ NK cell degranulation assay}

176 Murine NK cells were incubated for $2 \mathrm{~h}$ in the presence of GABA (2 or $10 \mu \mathrm{M}$, Sigma-Aldrich), 177 supernatants from unchallenged mBMDCs or supernatants from mBMDCs challenged with $T$. 178 gondii (PTG, MOI2, $24 \mathrm{~h}$ ). Supernatants were added at a medium:supernatant volume ratio of 179 1:1. NK cells were then washed and co-cultured with YAC-1 cells at a ratio of 10:1 in the 180 presence of anti-CD107a antibody (LAMP-1, eBioscience) diluted 1:200) The cells were briefly 181 centrifuged and after 30 min, monensin (BioLegend) was added to the culture for further $1.5 \mathrm{~h}$. 182 After this time point, cells were stained for CD3 (clone 145-2C11, Biolegend), NK1.1 (PK136, 183 Biolegend) and fixable viability dye (eFluor 780, eBioscience). Flow cytometry was performed on CyAN ADP LX 9-colour flow cytometer (Beckman Coulter, Pasadena, CA).

\subsection{Cytotoxicity assay}

187 Human NK-92 cells were preincubated with GABA for $2 \mathrm{~h}$ prior to use in the cytotoxicity assay and washed prior to use in the cytotoxicity assay. K-562 cells were incubated for $1 \mathrm{~h}$ in the presence of $\mathrm{Na}_{2}{ }^{51} \mathrm{CrO}_{4}\left({ }^{51} \mathrm{Cr}\right.$, Perkin Elmer), washed twice, and incubated with NK-92 cells at the indicated effector:target (E:T) ratios. After $2 \mathrm{~h}$, cell culture supernatants were collected and analyzed by a radiation counter (Wallac, PerkinElmer). Specific lysis was calculated as follows: \% specific lysis [(experimental release $\mathrm{x}$ spontaneous release) / (maximum release $\mathrm{x}$ spontaneous release)] $\times 100$.

\subsection{Western blot}

196 Human NK cells were challenged with freshly-egressed T. gondii tachyzoites (RH-LDM, MOI 1972,24 h). Cells were harvested and lysed in RIPA buffer with protease inhibitor cocktail 198 (Roche), sonicated, diluted with 4X Laemmli buffer and boiled. Samples were subjected to 199 SDS-PAGE on 8\% polyacrylamide gels, transferred onto PVDF membrane (Hybond P, Merck 200 Millipore), blocked in 5\% BSA for $30 \mathrm{~min}$ and incubated ON with mouse anti-GAD67 201 monoclonal antibody (1:6000, MAB5406, Merck Millipore) or $1 \mathrm{~h}$ with rabbit polyclonal anti202 GAPDH antibody (1:3000, ABS16, Merck Millipore) followed by incubation with respective 203 HRP-conjugated secondary antibodies (G21040, Thermofisher; 7074S, Cell signaling). Protein 
204 bands were revealed by enhanced chemiluminescence reagents (ECL ${ }^{\mathrm{TM}}$ Prime, Merck

205 Millipore) in a ChemiDoc XRS+ imaging system (BioRad, Stockholm, Sweden). Mouse brain

206 protein was used as positive control.

$208 \quad 2.11$ Statistical analyses

209 Data mining and statistical analyses were performed using GraphPad Prism 7.0 (La Jolla, CA,

210 USA) and described in figure legends. Statistical significance was defined as $p<0.05$.

211

$212 \quad 2.12$ Online Supplemental material

213 Supplementary Table 1. Primer pair sequences used in real-time quantitative PCR

214 Supplementary Table 2. Transcriptional expression of GABA-A R subunits, GABA enzymes,

215 GABA transporters and CCCs in T. gondii-challenged primary mNK cells

216 Supplementary Table 3. Transcriptional expression of GABA-A R subunits, GABA enzymes,

217 GABA transporters and CCCs in $T$. gondii-challenged primary hNK cells

218 Supplementary Table 4. Correlation analyses of mRNA expression levels with amounts of

219 GABA released by $T$. gondii-challenged NK cells from individual human donors

220 Supplementary Figure 1. Representative flow cytometric analysis of cell purity of mNK cells

221 used for mRNA analyses of GABA-related genes

222 Supplementary Figure 2. Relative mRNA expression of 19 GABA-A R subunits in NK cells

223 from four different human donors.

\section{Results}

\section{3.1 Murine and human NK cells transcribe a GABAergic machinery}

227 Whether NK cells express a GABAergic machinery has remained elusive. We therefore assessed

228 mouse and human NK cells for transcripts of GABA signaling components. Interestingly, both

229 murine and human NK cells expressed a number of transcripts of GABA-A receptors (GABA-A R)

230 subunits (Fig. 1A, Supplementary Fig. 2). Commonly expressed GABA-A R subunits for mouse

231 and human NK cells were $\alpha 3, \beta 2-3, \gamma 1, \delta$ and $\rho 1-2$, while additional subunits were not amplified

232 or only expressed in mouse or in human NK cells (Table 1). Furthermore, NK cells expressed

233 transcripts of the GABA synthesis enzymes glutamate decarboxylase (GAD65/67), GAD67 being

234 the most prominently expressed, and also the degrading enzyme GABA transaminase (GABA-T) 
235 (Fig. 1B). Interestingly, among known GABA transporters, only transcripts of GAT2 were

236 detected, similarly in mouse and human NK cells (Fig. 1C). Finally, a number of cation-chloride

237 cotransporters (CCC), known to regulate GABA signaling, were transcribed in both cell types

238 (Fig. 1D). We conclude that human and mouse NK cells transcribe a set of GABAergic signaling

239 components that, in theory, suffice to synthesize, degrade and transport GABA, and form

240 functional GABA-A Rs.

241

$242 \quad 3.2$ Transcriptional modulation of GABAergic components in NK cells upon challenge with $T$. 243 gondii

244 Next, we addressed if challenge of NK cells with $T$. gondii tachyzoites impacted the 245 transcriptional expression of GABAergic signaling components. We previously reported that $T$. 246 gondii actively invades and replicates within NK cells ${ }^{20}$. Overall, a transcriptional upregulation 247 was observed for multiple GABAergic components (Fig. 2, Supplementary Tables 2 and 3). 248 Interestingly, up and downregulations were observed for GABA-A R subunits (Fig. 2A) and the 249 GABA regulators CCCS (Fig. 2D), indicating a significant impact of infection on their expression. 250 In contrast, the transcription of GABA synthesis enzymes (Fig. 2B) and transporters (Fig. 2C) were consistently upregulated, with the exception of the GABA degrading enzyme GABA-T in

252 human NK cells. We conclude that infection challenge led to upregulated expression of multiple 253 GABAergic signaling components in NK cells.

\subsection{T. gondii-infection induces GABA synthesis and secretion by human and murine NK cells}

256 Next, we sought to determine if NK cells produce and secrete GABA. First, in T. gondii257 challenged NK cells from human donors, we confirmed the presence of protein bands 258 corresponding to the synthesis enzyme GAD67 from mouse brain homogenates (Fig. 3A), 259 consistent with the observed transcriptional upregulation of GAD67 mRNA (Fig. 2B). 260 Importantly, upon challenge with T. gondii, GABA-ELISA analyses revealed a dramatic elevation 261 of GABA in the supernatant for both mouse and human NK cells (Fig. 3B, C). In presence of the 262 GAD-inhibitor semicarbazide (SC), the amounts of GABA in the supernatant were significantly 263 reduced (Fig. 3D). Moreover, MOl-equivalent doses of heat-inactivated tachyzoites, tachyzoite 264 lysates and supernatants or addition of cytokines failed to induce GABA secretion (Fig. 3D), 265 indicating that live intercellular parasites were necessary for GABAergic activation. Next, we 266 explored GABA responses by individual human donors. NK cells from 9 human donors 
267

268

269

270

271

272

273

274

275

276

277

278

279

280

281

282

283

284

285

286

287

288

289

290

291

292

293

294

295

296

297

298

consistently responded to $T$. gondii challenge with elevated GABA secretion, and with variations among donors in the amounts of secreted GABA (Fig. 3E). This variation motivated an analysis of the expression of the GABA synthesis enzyme GAD67 and the GABA catabolic enzyme GABAT for each donor. Interestingly, the amounts of secreted GABA by the individual donors were strongly correlated with an upregulation of GAD67 and downregulation of GABA-T expression, respectively (Fig. 3F). This correlation was absent for GABAergic genes unrelated to GABA metabolism (Supplementary Table 4). Finally, T. gondii-challenged NK cells were sorted in infected cells and by-stander cells (Fig. 3G). Supernatants from sorted infected NK cells presented significantly elevated GABA production related to supernatants from by-stander cells (Fig 3H). However, GABA concentrations were elevated in supernatants from by-stander cells related to supernatants from unchallenged cells (Fig $\mathbf{3 H}$ ), indicating a minor by-stander effect, and with variations among human donors (Fig 31). Jointly, the data demonstrate that NK cells synthesize and secrete GABA, which is dramatically accentuated upon T. gondii infection. Moreover, the data are indicative that the elevated amounts of secreted GABA by $T$. gondiiinfected NK cells are mediated by increased GABA synthesis (GAD67) and decreased GABA catabolism (GABA-T).

\subsection{Exogenous GABA and supernatants from T. gondii-infected DCs hamper NK cell} degranulation and cytotoxicity

Previously, we found that murine NK cells infected with $T$. gondii exhibit impaired cytotoxicity towards YAC-1, the prototypic NK cell target cell ${ }^{30}$. To test if GABA impacted this central effector function of NK cells, degranulation and cytotoxicity assays were performed in the presence of exogenous GABA at a concentration range corresponding to that attained in supernatants of DCs challenged with $T$. gondii ${ }^{3}$ and challenged NK cells (low $\mu \mathrm{M}$ range) (Fig. 3). In presence of GABA, NK cells consistently exhibited reduced degranulation (Fig. 4A, B), indicating that GABA had a negative impact on NK cell-mediated cytotoxicity in the presence of YAC-1 cells. Next, we tested if GABA impacted the cytotoxicity of NK-92 cells, a human NK cell line. Similar to the experiments with the mouse NK cells, GABA inhibited NK-92 cell-mediated killing of K-562 cells (Fig. 4C), confirming that GABA inhibits NK cell cytotoxicity in vitro. Finally, we tested GABA-containing supernatant from T. gondii-challenged DCs. While there was a nonsignificant difference between mouse IL-2-stimulated NK cells pre-treated with supernatant from infected DCs compared with control supernatant, the overall trend was that the 
299

300

301

302

303

304

305

306

307

308

309

310

311

312

313

314

315

316

317

318

319

320

321

322

323

324

325

326

327

328

329

supernatants from infected DCs had a negative impact on degranulation by NK cells in the presence of YAC-1 cells (Fig. 4D, E). Jointly, the data indicate that GABA reduces NK cell cytotoxicity in vitro.

\subsection{Supernatants from $T$. gondii-challenged NK cells rescue hypermotility in GABA-inhibited} parasitized DCs

GABAergic signaling drives the migratory activation of parasitized DCs, termed hypermotility ${ }^{3}$. We previously reported that transfer of $T$. gondii from parasitized DCs to effector NK cells leads to productive infection of NK cells ${ }^{20}$. However, whether NK-DC interactions can be modulated by GABAergic signaling has not been addressed. We therefore sought to determine if supernatants from infected NK cells could modulate the motility of parasitized DCs. First, DCs where subjected to GABAergic inhibition with the GABA synthesis inhibitor SC, which abrogated hypermotility of infected DCs, with a non-significant impact on base-line motility of unchallenged DCs (Fig. 5A, B). Importantly, GABA-containing supernatant collected from parasitized NK cells (Fig. 3D), restored hypermotility of parasitized DCs in presence of SC (Fig 5A, B). This reconstitution was abolished by the broad GABA-A $R$ inhibitor picrotoxin, suggesting an indispensable role for GABA-A Rs in hypermotility of DCs. Further, supernatant from NK cells pretreated with SC (Fig. 3C) failed to reconstitute hypermotility. Finally, addition of exogenous GABA reconstituted hypermotility in presence of SC. We conclude that GABAcontaining NK cell supernatants rescue hypermotility of parasitized DCs. Consistent with the GABAergic signaling cascade ${ }^{36}$, GABA-containing NK cell supernatants rescued hypermotility when upstream GABA synthesis was inhibited (SC) but not when downstream GABA-A R activation was inhibited (picrotoxin). Thus, GABA-containing supernatants from NK cells impacted the motility of parasitized DCs in a picrotoxin inhibitable fashion which was rescued by exogenous GABA, jointly indicating implication of GABA-A Rs.

\section{Discussion}

To date, the GABAergic potential of NK cells has remained unexplored ${ }^{11}$. Here, we report that NK cells are in fact GABAergic cells and that GABA signaling is implicated in the responses of NK cells challenged with $T$. gondii. Our study establishes that both human and murine NK cells transcriptionally express components of the GABAergic system including GABA 
330 synthesis and degradation enzymes, GABA transporters, GABA-A R subunits and regulators

331 (CCCS). However, the overall expression repertoire of GABA-A R subunits was more restricted in

332 NK cells compared with primary microglia ${ }^{34}$ and more similar to that of DCs ${ }^{3}$. Commonly 333 expressed GABA-A R subunits for human and mouse NK cells were $\alpha 3, \beta 2, \gamma 1$ and $\rho 1 / 2$. This

334 expression repertoire in NK cells provides a base for the formation of both heteropentameric ( 2

$335 \alpha: s+2 \beta: s+1$ additional subunit) and homopentameric ( $\rho: s)$ GABA-A Rs. Further, in human NK

336 cells, variability in the expressed subunits was observed among donors. Yet, the $\alpha 3, \beta 2$ and $\rho 2$

337 subunits were commonly expressed by all tested donors. Jointly, this indicates a conserved

338 expression of $\alpha, \beta$ and $\rho$ subunits for human and murine NK cells.

$339 \mathrm{Cl}^{-}$transporters (CCCs) regulate the level of intracellular $\mathrm{Cl}^{-}$and ultimately govern the

340 effects of GABA-A R activation ${ }^{6}$. The expression pattern of CCCS in NK cells was consistent with

341 that found in peripheral blood mononuclear cells ${ }^{12}$ and microglia ${ }^{34}$. The CCCs NKCC1 and KCC2

342 are major regulators of intracellular $\mathrm{Cl}^{-}$concentrations in neuronal cells ${ }^{37}$. Because expression

343 of KCC2 was low or absent in NK cells, NKCC1 is likely the principal determinant for setting the

344 intracellular $\mathrm{Cl}^{-}$concentration and thus, for regulating the effects mediated by GABA-A R 345 activation.

346 In GABAergic mammalian cells, GABA is chiefly synthesized from glutamate by the 347 action of the enzymes GAD65 and GAD67 ${ }^{7}$. GAD65 synthesizes GABA mainly for vesicular 348 release and GAD67 for cytosolic release. The expression of GAD67 by peripheral immune cells 349 has remained undetermined. Here, we show that murine NK cells transcribe both enzymes, 350 similar to murine microglia ${ }^{34}$. In contrast, our data indicate that human NK cells exclusively 351 transcribe GAD67 and express GAD67 protein upon T. gondii challenge. Initially, murine T cells, 352 macrophages and DCs were reported to express GAD65 and secrete measurable amounts of $353 \mathrm{GABA}^{3,13}$. However, a more recent report characterized the expression of GAD65/67 in murine 354 DCs, while human monocytes and DCs exclusively expressed GAD67 ${ }^{38}$. Thus, expression of 355 GAD67 over GAD65 seems to distinguish human NK cells, and human mononuclear phagocytes, 356 from other peripheral immune cells. Regardless, the data demonstrate abundant release of 357 GABA by both human and murine NK cells upon challenge with $T$. gondii. The upregulation of 358 GAD transcripts likely explains the elevated GABA secretion upon infection, which was inhibited 359 by GAD inhibition. Jointly, this imparts functional roles for GADs in NK cells. 

and DCs ${ }^{3,34}$ and GAT1 was dysregulated in T cells in models of multiple sclerosis and experimental autoimmune encephalomyelitis, impacting proliferative and cytotoxic immune responses ${ }^{13,39}$. Our assumption that GAT2 transports GABA out of NK cells for autocrine stimulation of GABA A Rs is consistent with attributed functions of efflux of GABA by GAT2 in neuronal models, including release of GABA across the blood-brain barrier ${ }^{40}$. Taken together, the expression of GABAergic signaling components in NK cells and the implication of the GABAergic system of T cells and phagocytes in immunomodulation and responses to infection ${ }^{41}$ motivated a functional assessment of GABAergic signaling in NK cells.

We show that GABAergic activation has functional implications for NK cell effector functions and interactions with DCs. Importantly, NK cells responded to $T$. gondii infection with GABA secretion. Jointly with GAD67, the expression of GAT2 supports the notion that GABA is synthesized cytosolically and secreted in vesicle-independent fashion for tonic modulations of GABA-A Rs in NK cells, similar to neurons ${ }^{40,42}$. However, exactly how the elevated GABA synthesis and secretion is orchestrated in NK cells remains to be investigated. Nevertheless, the consistent reciprocal upregulation of the GABA synthesis enzyme and downregulation of GABAdegrading GABA-T in distinct human donors is indicative that GABA production is tightly regulated in NK cells. In DCs and primary microglia, GABA production requires live intracellular T. gondii ${ }^{3,34}$. Similarly, NK cells responded with transcriptional modulation of several components of the GABAergic system upon infection by $T$. gondii. Cell sorting revealed that GABA was chiefly secreted by infected $\left(\mathrm{GFP}^{+}\right)$NK cells, corroborating the association to intracellular localization of live parasites. However, a modest but significant by-stander effect was consistently detected in non-infected (GFP) NK cells, which could not be achieved by challenge with heat-inactivated parasites, lysates, supernatants or IL12/IL18 activation. We speculate that this by-stander effect may be mediated by injection of parasite effectors in host cells in absence of invasion ${ }^{43}$. Altogether, secretion of GABA, upregulation of GABA transporters, upregulation of GABA-A R subunits and GABA signaling regulators CCCs represent an enhanced GABAergic signaling in NK cells upon infection.

We report that GABA downmodulates cytotoxicity and degranulation of NK cells in vitro.

390 Previous work has shown that NK cells target parasitized DCs and that cytotoxic attack can lead

391 to infection of NK cells ${ }^{20}$. Interestingly, cytokine responses, and specifically IFN- $\gamma$ responses, are 
392 downmodulated in parasitized NK cells by unknown mechanisms ${ }^{30}$. Previous studies have 393 shown that GABA can regulate cytokine release by peripheral blood mononuclear cells and T 394 cells ${ }^{44}$, and T cell cytotoxicity ${ }^{45}$ but it has remained unclear what effects GABA has on NK cells

$395{ }^{46}$. Here, we show that GABA, which is secreted by parasitized NK cells and DCs, hampers 396 cytotoxicity and degranulation of NK cells in vitro. Additionally, secreted GABA also modulated 397 the migratory responses of DCs, shown by the reconstitution of hypermotility in parasitized 398 GABA-inhibited DCs by GABA-containing supernatants from parasitized NK cells or exogenous 399 GABA, but not upon GABA-A R antagonism. Altogether, we speculate that GABA may have dual 400 effects during infection: down-modulation of pro-inflammatory responses and enhancement of 401 DC migration. Hypothetically, in the context of infection in the microenvironment in tissues, this 402 dual effect might facilitate transmission of the parasite between leukocytes and thus facilitate 403 dissemination. However, in contrast to DCs, the migratory responses of parasitized NKs in vitro 404 seem to be non-significantly affected ${ }^{47}$. Because both DCs and NK cells secrete GABA upon $T$. 405 gondii infection, the different migratory responses of the two cell types indicate differences in 406 GABA-A R function. This is also consistent with the present findings that DCs and NKs express 407 different subsets of GABA-A R subunits.

408 To our knowledge, this represents the first report of GABAergic signaling in NK cells. The 409 data provide a proof-of-concept that GABAergic signaling can modulate NK cell functions in the 410 context of infection. We have recently shown that $T$. gondii infection impacts MAP kinase 411 activation and calcium signaling in immune cells ${ }^{4,36,48}$. Thus, immunomodulatory effects in 412 parasitized NK, for example cytokine secretion, could be mediated through MAP kinase 413 signaling. If GABAergic signaling acts on NK cells via voltage-gated calcium channels awaits 414 further investigation. Taking into account recent reports showing that T cells, DCs, monocytes, 415 macrophages and microglia are GABAergic cells ${ }^{34,36,44}$, the mounting evidence advocates that 416 GABAergic signaling may be conserved throughout the immune system. GABAergic activation in 417 immune cells is likely not restricted to intracellular parasitism and, hypothetically, encompasses 418 additional immune cell functions.

420 Authorship. AKB, LMF, SK, SJ-B, IE-L, AKW, BJC performed experimental work. AKB, AKW, BJC 421 and $A B$ analyzed the data. AKW and BJC contributed human and murine materials. AKB, BJC and 422 AB prepared figures and wrote manuscript. 
424 Acknowledgements. This work was funded by the Swedish Research Council (Vetenskapsrådet,

425 2018-02411 to $A B, 2017-00923$ to $B J C$ ), the Olle Engkvist Foundation (193-609 to $A B$ ) and

426 Cancerfonden (CAN 2017/714 to BJC).

427

428 Conflict of interest disclosure. Authors declare that research was conducted in the absence of

429 any commercial or financial relationships that could be construed as a potential conflict of

430 interest.

431

432 References

4331 Azuma, $\mathrm{H}$. et al. Gamma-aminobutyric acid as a promoting factor of cancer 434 metastasis; induction of matrix metalloproteinase production is potentially its underlying mechanism. Cancer Res 63, 8090-8096 (2003).

2 Wheeler, D. W. et al. Anaesthetic impairment of immune function is mediated via

437

438 GABA(A) receptors. PloS one 6, e17152, doi:10.1371/journal.pone.0017152 (2011).

3 Fuks, J. M. et al. GABAergic signaling is linked to a hypermigratory phenotype in 439 dendritic cells infected by Toxoplasma gondii. PLoS pathogens 8, e1003051, doi:10.1371/journal.ppat.1003051 (2012).

442

443

444

445

4 Kanatani, S. et al. Voltage-dependent calcium channel signaling mediates GABAA gondii. PLoS pathogens 13, e1006739, doi:10.1371/journal.ppat.1006739 (2017).

5 Olsen, R. W. \& Sieghart, W. International Union of Pharmacology. LXX. Subtypes of gamma-aminobutyric acid(A) receptors: classification on the basis of subunit composition, pharmacology, and function. Update. Pharmacol Rev 60, 243-260, doi:10.1124/pr.108.00505 (2008).

451

452

453

454

455

456

457

458 Kahle, K. T. et al. Roles of the cation-chloride cotransporters in neurological disease.

Nat Clin Pract Neurol 4, 490-503, doi:10.1038/ncpneuro0883 (2008).

7 Soghomonian, J. J. \& Martin, D. L. Two isoforms of glutamate decarboxylase: why? Trends in pharmacological sciences 19, 500-505 (1998).

8 Hoglund, P. J., Adzic, D., Scicluna, S. J., Lindblom, J. \& Fredriksson, R. The repertoire of solute carriers of family 6: identification of new human and rodent genes. Biochem Biophys Res Commun 336, 175-189, doi:10.1016/j.bbrc.2005.08.048 (2005).

9 Kilb, W. Development of the GABAergic system from birth to adolescence. Neuroscientist 18, 613-630, doi:10.1177/1073858411422114 (2012).

10 Lee, M., Schwab, C. \& McGeer, P. L. Astrocytes are GABAergic cells that modulate microglial activity. Glia 59, 152-165, doi:10.1002/glia.21087 (2011).

11 Barragan, A., Weidner, J. M., Jin, Z., Korpi, E. R. \& Birnir, B. GABAergic signalling in the immune system. Acta physiologica 213, 819-827, doi:10.1111/apha.12467 (2015).

461

462

12 Bhandage, A. K. et al. Expression of GABA receptors subunits in peripheral blood mononuclear cells is gender dependent, altered in pregnancy and modified by mental health. Acta physiologica 213, 575-585, doi:10.1111/apha.12440 (2015). 
465

466

467

468

469

470

471

472

473

474

475

476

477

478

479

480

481

482

483

484

485

486

487

488

489

490

491

492

493

494

495

496

497

498

499

500

501

502

503

504

505

506

507

508

509

510

13 Bhat, R. et al. Inhibitory role for GABA in autoimmune inflammation. Proceedings of the National Academy of Sciences of the United States of America 107, 2580-2585, doi:0915139107 [pii]

10.1073/pnas.0915139107 (2010).

14 Tenter, A. M., Heckeroth, A. R. \& Weiss, L. M. Toxoplasma gondii: from animals to humans. Int. J. Parasitol. 30, 1217-1258 (2000).

15 Schluter, D. \& Barragan, A. Advances and Challenges in Understanding Cerebral Toxoplasmosis. Front Immunol 10, 242, doi:10.3389/fimmu.2019.00242 (2019).

16 Montoya, J. G. \& Liesenfeld, O. Toxoplasmosis. Lancet 363, 1965-1976 (2004).

17 Frenal, K., Dubremetz, J. F., Lebrun, M. \& Soldati-Favre, D. Gliding motility powers invasion and egress in Apicomplexa. Nature reviews 15, 645-660, doi:10.1038/nrmicro.2017.86 (2017).

18 Lambert, H., Hitziger, N., Dellacasa, I., Svensson, M. \& Barragan, A. Induction of dendritic cell migration upon Toxoplasma gondii infection potentiates parasite dissemination. Cell Microbiol 8, 1611-1623, doi:10.1111/j.1462-5822.2006.00735.x (2006).

19 Persson, E. K. et al. Death receptor ligation or exposure to perforin trigger rapid egress of the intracellular parasite Toxoplasma gondii. J Immunol 179, 8357-8365 (2007).

20 Persson, C. M. et al. Transmission of Toxoplasma gondii from infected dendritic cells to natural killer cells. Infect Immun 77, 970-976, doi:10.1128/IAI.00833-08 (2009).

21 Olafsson, E. B. \& Barragan, A. The unicellular eukaryotic parasite Toxoplasma gondii hijacks the migration machinery of mononuclear phagocytes to promote its dissemination. Biol Cell, doi:10.1111/boc.202000005 (2020).

22 Gigley, J. P. The Diverse Role of NK Cells in Immunity to Toxoplasma gondii Infection. PLoS pathogens 12, e1005396, doi:10.1371/journal.ppat.1005396 (2016).

23 Denkers, E. Y., Gazzinelli, R. T., Martin, D. \& Sher, A. Emergence of NK1.1+ cells as effectors of IFN-gamma dependent immunity to Toxoplasma gondii in MHC class Ideficient mice. The Journal of experimental medicine 178, 1465-1472, doi:10.1084/jem.178.5.1465 (1993).

24 Sher, A., Oswald, I. P., Hieny, S. \& Gazzinelli, R. T. Toxoplasma gondii induces a Tindependent IFN-g response in natural killer cells that requires both adherent accessory cells and tumor necrosis factor-a. Journal of Immunology 150, 3982-3989 (1993).

25 Hunter, C. A., Subauste, C. S., Van Cleave, V. H. \& Remington, J. S. Production of gamma interferon by natural killer cells from Toxoplasma gondii-infected SCID mice: regulation by interleukin-10, interleukin-12, and tumor necrosis factor alpha. Infection and immunity 62, 2818-2824 (1994).

26 Combe, C. L., Curiel, T. J., Moretto, M. M. \& Khan, I. A. NK cells help to induce CD8(+)-T-cell immunity against Toxoplasma gondii in the absence of CD4(+) T cells. Infection and immunity 73, 4913-4921, doi:10.1128/IAI.73.8.4913-4921.2005 (2005).

27 Guan, H., Moretto, M., Bzik, D. J., Gigley, J. \& Khan, I. A. NK cells enhance dendritic cell response against parasite antigens via NKG2D pathway. J Immunol 179, 590-596, doi:10.4049/jimmunol.179.1.590 (2007).

28 Coombes, J. L., Han, S. J., van Rooijen, N., Raulet, D. H. \& Robey, E. A. Infectioninduced regulation of natural killer cells by macrophages and collagen at the lymph 
511

512

513

514

515

516

517

518

519

520

521

522

523

524

525

526

527

528

529

530

531

532

533

534

535

536

537

538

539

540

541

542

543

544

545

546

547

548

549

550

551

552

553

554

555

556

node subcapsular sinus. Cell Rep 2, 124-135, doi:10.1016/j.celrep.2012.06.001 (2012).

29 Ueno, N., Lodoen, M. B., Hickey, G. L., Robey, E. A. \& Coombes, J. L. Toxoplasma gondii-infected natural killer cells display a hypermotility phenotype in vivo. Immunology and cell biology 93, 508-513, doi:10.1038/icb.2014.106 (2015).

30 Sultana, M. A. et al. Downmodulation of Effector Functions in NK Cells upon Toxoplasma gondii Infection. Infection and immunity 85, doi:10.1128/IAl.00069-17 (2017).

31 Mombaerts, P. et al. RAG-1-deficient mice have no mature B and T lymphocytes. Cell 68, 869-877, doi:10.1016/0092-8674(92)90030-g (1992).

32 Kim, K., Eaton, M. S., Schubert, W., Wu, S. \& Tang, J. Optimized expression of green flourescent protein in Toxoplasma gondii using thermostable green flourescent protein mutants. Mol. Biochem. Parasitol. 113, 309-313 (2001).

33 Hitziger, N., Dellacasa, I., Albiger, B. \& Barragan, A. Dissemination of Toxoplasma gondii to immunoprivileged organs and role of Toll/interleukin-1 receptor signalling for host resistance assessed by in vivo bioluminescence imaging. Cellular microbiology 7, 837-848, doi:CMI517 [pii]

10.1111/j.1462-5822.2005.00517.x (2005).

34 Bhandage, A. K., Kanatani, S. \& Barragan, A. Toxoplasma-Induced Hypermigration of Primary Cortical Microglia Implicates GABAergic Signaling. Front Cell Infect Microbiol 9, 73, doi:10.3389/fcimb.2019.00073 (2019).

35 Weidner, J. M. et al. Rapid cytoskeleton remodelling in dendritic cells following invasion by Toxoplasma gondii coincides with the onset of a hypermigratory phenotype. Cellular microbiology 15, 1735-1752, doi:10.1111/cmi.12145 (2013).

36 Bhandage, A. K. et al. A motogenic GABAergic system of mononuclear phagocytes facilitates dissemination of coccidian parasites. Elife 9, doi:10.7554/eLife.60528 (2020).

37 Kaila, K., Price, T. J., Payne, J. A., Puskarjov, M. \& Voipio, J. Cation-chloride cotransporters in neuronal development, plasticity and disease. Nat Rev Neurosci 15, 637-654, doi:10.1038/nrn3819 (2014).

38 Bhandage, A. K. et al. Expression of GABA receptors subunits in peripheral blood mononuclear cells is gender dependent, altered in pregnancy and modified by mental health. Acta physiologica, doi:10.1111/apha.12440 (2014).

39 Wang, Y. et al. Gamma-aminobutyric acid transporter 1 negatively regulates T cellmediated immune responses and ameliorates autoimmune inflammation in the CNS. $\mathrm{J}$ Immunol 181, 8226-8236, doi:10.4049/jimmunol.181.12.8226 (2008).

40 Takanaga, H., Ohtsuki, S., Hosoya, K. \& Terasaki, T. GAT2/BGT-1 as a system responsible for the transport of gamma-aminobutyric acid at the mouse blood-brain barrier. J Cereb Blood Flow Metab 21, 1232-1239, doi:10.1097/00004647200110000-00012 (2001).

41 Jin, Z., Mendu, S. K. \& Birnir, B. GABA is an effective immunomodulatory molecule. Amino Acids 45, 87-94, doi:10.1007/s00726-011-1193-7 (2013).

42 Kaufman, D. L., Houser, C. R. \& Tobin, A. J. Two forms of the gamma-aminobutyric acid synthetic enzyme glutamate decarboxylase have distinct intraneuronal distributions and cofactor interactions. J Neurochem 56, 720-723, doi:10.1111/j.1471-4159.1991.tb08211.x(1991). 
$55743 \quad$ Koshy, A. A. et al. Toxoplasma co-opts host cells it does not invade. PLoS pathogens 8, e1002825, doi:10.1371/journal.ppat.1002825 (2012).

559

44 Bhandage, A. K. et al. GABA Regulates Release of Inflammatory Cytokines From

560 Peripheral Blood Mononuclear Cells and CD4(+) T Cells and Is Immunosuppressive in Type 1 Diabetes. EBioMedicine 30, 283-294, doi:10.1016/j.ebiom.2018.03.019

562 (2018).

563

45 Bergeret, M. et al. GABA modulates cytotoxicity of immunocompetent cells

564

565 expressing GABAA receptor subunits. Biomed Pharmacother 52, 214-219, doi:10.1016/S0753-3322(98)80019-X (1998).

567

46 Lang, K., Drell, T. L., Niggemann, B., Zanker, K. S. \& Entschladen, F. Neurotransmitters

568 regulate the migration and cytotoxicity in natural killer cells. Immunol Lett 90, 165-

569 172, doi:10.1016/j.imlet.2003.09.004 (2003).

570

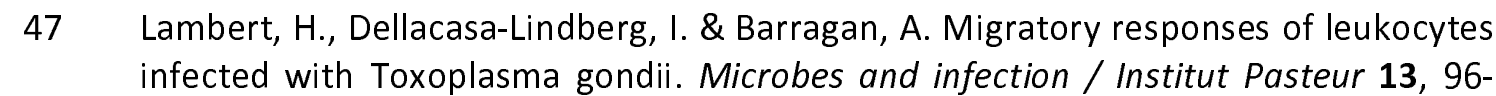
102, doi:10.1016/j.micinf.2010.10.002 (2011).

572

48 Olafsson, E. B. et al. Convergent Met and voltage-gated $\mathrm{Ca}(2+)$ channel signaling drives hypermigration of Toxoplasma-infected dendritic cells. Journal of cell science 134, doi:10.1242/jcs.241752 (2020). 


\section{$577 \quad$ Figure legends}

578 Figure 1. Expression of GABA signaling components by murine and human NK cells

579 Bar graphs show relative mRNA expression $\left(2^{-\Delta C t}+S E M\right)$ of (A) GABA-A R subunits, (B) GABA

580 synthesis and catabolic enzymes (GAD65/67 and GABA-T, respectively), (C) GABA

581 transporters and (D) cation-chloride co-transporters (CCCs) in mouse NK cells (mNK, $n=8$

582 mice) and human NK cells (hNK, $n=4$ human donors).

583

584 Figure 2. Transcriptional modulation in GABA signaling components in NK cells upon $T$. 585 gondii challenge

586 (A-D) Heat maps show transcriptional changes of indicated GABA signaling components 587 upon challenge of murine NK cells (mNK) and human NK cells (hNK) with T. gondii (RH-LDM), 588 at indicated time points. Red and blue color scales indicate, for each gene, the percentage 589 increase or decrease in expression, respectively, normalized to unchallenged cells at the 590 same time point. (X) indicates no amplification. Detailed numeric analyses of murine and 591 human NK cells are provided in Supplementary Tables 2 and 3, respectively ( $n=3-5$ 592 independent experiments).

593

\section{Figure 3. GABA secretion by human and murine NK cells}

595 (A) Representative Western blot of lysates from unchallenged and T. gondii-challenged NK 596 cells from 2 human donors (hNK1 and 2, respectively), immunoblotted with GAD67 antibody 597 as indicated under Methods. Mouse brain lysate $(\mathrm{mBr})$ served as a positive control and 598 GAPDH immunoblotting was performed for loading reference. (B, C) Bar graphs show 599 concentrations (mean +SEM) of GABA secreted in supernatants of (B) mouse NK cells (mNK, $600 \mathrm{n}=6$ mice) and (C) human NK cells ( $\mathrm{hNK}, \mathrm{n}=9$ human donors), respectively, challenged with 601 T. gondii (RH-LDM) and quantified by ELISA. ${ }^{* *} \mathrm{p}<0.01$, Mann-Whitney test. (D) GABA 602 concentration in mNK supernatants after $24 \mathrm{~h}$ of challenge with $T$. gondii (MOI 2 ) in 603 presence and absence of GABA synthesis inhibitor (SC), or with MOI-equivalent amounts of 604 heat-inactivated (HI) T. gondii, lysate (sonicated T. gondii), supernatants from T. gondii, or 605 cytokine stimulation with IL-12 and IL-18, as detailed in Methods. ns: non-significant, * $p<$ 6060.05 , one-way ANOVA followed by Tukey multiple comparison test. (E) GABA concentrations 607 upon T. gondii-challenge of NK cells from individual human donors (1-9). (F) Correlation 
608 analyses of transcriptional expression changes of GABA metabolic enzymes with measured

609 GABA concentrations in supernatants, for 9 human donors. For each donor, GABA

610 concentration in the supernatants of hNK cells was correlated with transcriptional changes

611 in GAD67 and GABA-T upon T. gondii challenge. Correlation was assessed by non-parametric

612 spearman test. $R^{2}$ indicates the linearity of the trend line. (G) Bivariate contour plots of hNK

613 cells challenged with GFP-expressing T. gondii (RH-LDM) and stained for CD56. GFP ${ }^{+}$CD56 $^{+}$

614 cells were defined as infected NK cells and GFP $\mathrm{CD}^{-} 6^{+}$cells as by-stander NK cells. (H) Bar

615 graph shows GABA concentrations in supernatants from cell-sorted unchallenged, by-

616 stander and infected hNK cells. $* p<0.05, * * * p<0.001$, one-way ANOVA followed by

617 Dunnett's multiple comparisons test ( $\mathrm{n}=3$ human donors). (I) GABA concentrations in

618 supernatants from cell-sorted by-stander and infected hNK cells from 3 human donors (1-3).

620 Figure 4. Degranulation of mouse NK cells and cytotoxicity of NK-92 cells in the presence of GABA

622 (A-B) NK cells were preincubated with GABA at indicated concentration for $2 \mathrm{~h}$ prior to 623 incubation with YAC-1 cells. (A) Representative bivariate plots show \% of cells with surface expression of NK1.1 and CD107a. (B) Box-and-whisker plots show median values from 6 independent experiments. ${ }^{* *} \mathrm{p}<0.01, * * * \mathrm{p}<0.001$, RM one-way ANOVA followed by

626 Dunnett's multiple comparison test, $(n=6$ independent experiments). (C) NK-92 cells were 627 incubated in the presence of GABA for $2 \mathrm{~h}$ prior to incubation with ${ }^{51} \mathrm{Cr}$ labelled K-562 cells.

628 Graph represents \% cytotoxicity calculated as indicated under Methods, * $p<0.05$, ** $p<$ 6290.01 , paired Student's $t$-test, $(n=4$ independent experiments with 3 technical replicates 630 each). (D, E) NK cells were incubated with supernatants from unchallenged mBMDCs or $T$. 631 gondii (RH-LDM)-infected mBMDCs for $2 \mathrm{~h}$ prior to incubation with YAC-1 cells for 632 measuring degranulation, as in (A-B). ns: $p>0.05$, paired Student's $t$-test, $(\mathrm{n}=4$ 633 independent experiments).

\section{Figure 5. Supernatants from infected NK cells induce hypermotility in mDCs}

636 (A) Representative motility plots of unchallenged and T. gondii (ME49/PTG)-infected 637 mBMDCs treated with SC in presence and absence of supernatants from $T$. gondii-infected $638 \mathrm{mNK}$ cells with or without picrotoxin. $\mathrm{X}$ - and $\mathrm{y}$-axes show distances in $\mu \mathrm{m}$. (B) Box-and639 whisker dot plots show cell velocities $(\mu \mathrm{m} / \mathrm{min})$ for each condition, as in $(A)$. Cells were 
640 treated with SC in presence of supernatants from T. gondii-infected mNK cells, picrotoxin or

641 GABA. Non-treated (NT) and SC-treated (SC) mNK cell supernatants were collected as

642 indicated under Methods and added at a 1:1 ratio with medium. $* * p<0.01, * * * p<0.001$,

643 ns: $p>0.05$, one-way ANOVA followed by Tukey multiple comparison test $(\mathrm{n}=3-6$

644 independent experiments per condition).

645 
Table 1. GABA-A Receptor subunits transcribed by NK cells

\begin{tabular}{|c|c|c|c|c|c|c|c|c|c|c|c|c|c|c|c|c|c|c|c|c|}
\hline Cell / tissue & Condition & $\alpha 1$ & $\alpha 2$ & $\alpha 3$ & $\alpha 4$ & $\alpha 5$ & $\alpha 6$ & $\beta 1$ & $\beta 2$ & $\beta 3$ & $\gamma 1$ & $\gamma 2$ & $\gamma^{3}$ & $\delta$ & $\varepsilon$ & $\theta$ & $\pi$ & $\rho 1$ & $\rho 2$ & 3 \\
\hline $\mathrm{mNK}$ & Unchallenged & & $6 / 8^{*}$ & $3 / 8$ & & & & & $6 / 8$ & $7 / 8$ & $7 / 8$ & $4 / 8$ & & $8 / 8$ & $7 / 8$ & & $3 / 8$ & $8 / 8$ & $4 / 8$ & \\
\hline hNK & Unchallenged & & & $4 / 4$ & & $4 / 4$ & $4 / 4$ & & $4 / 4$ & $1 / 4$ & $3 / 4$ & & & $3 / 4$ & & $3 / 4$ & & $3 / 4$ & $4 / 4$ & \\
\hline Mouse brain & Unchallenged & $4 / 4$ & $4 / 4$ & $4 / 4$ & $4 / 4$ & $4 / 4$ & $4 / 4$ & $4 / 4$ & $4 / 4$ & $4 / 4$ & $4 / 4$ & $4 / 4$ & $4 / 4$ & $4 / 4$ & $4 / 4$ & $4 / 4$ & $4 / 4$ & $4 / 4$ & $4 / 4$ & $4 / 4$ \\
\hline
\end{tabular}

Murine NK cells (mNK) and human NK cells (hNK) were assessed for mRNA expression of GABA-A receptor subunits after challenge with $T$. gondii as detailed under Methods. Mouse whole brain homogenate mRNA was used as reference material.

* indicates number of mice or human donors, respectively, expressing a particular subunit related to the total number of independent samples analyzed ( $\mathrm{mNK}, \mathrm{n}=8$ mice; $\mathrm{hNK}, \mathrm{n}=4$ human donors; mouse brain homogenate, $\mathrm{n}=4$ mice).

Blank spaces indicate that the particular subunit mRNA was not amplified in any sample. 

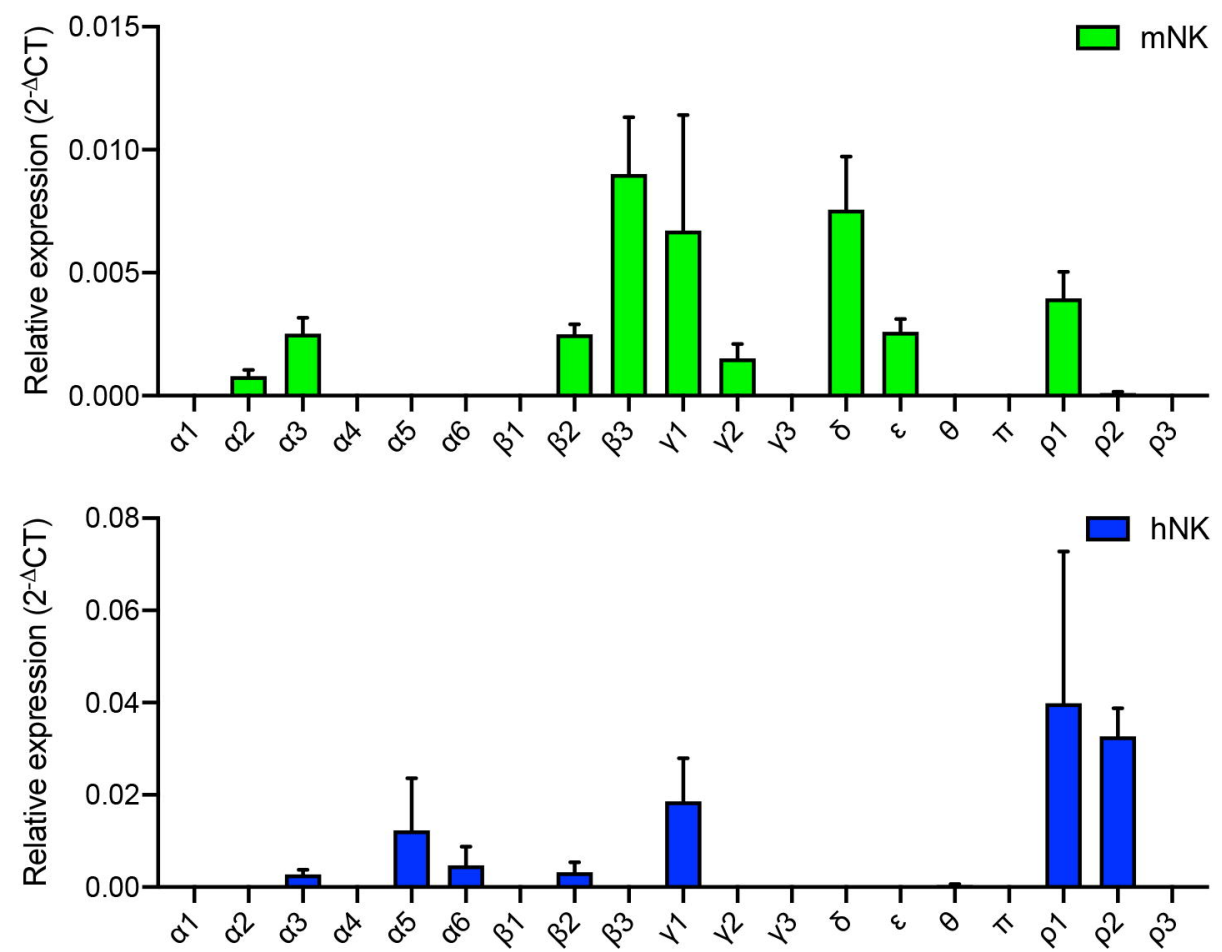

B
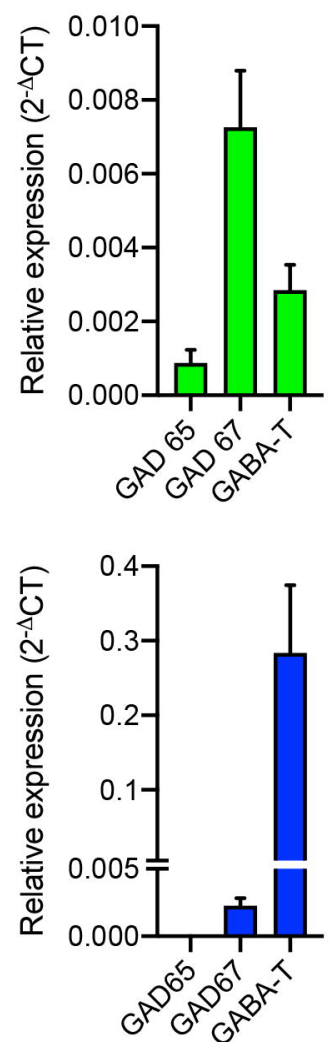

C Transporters
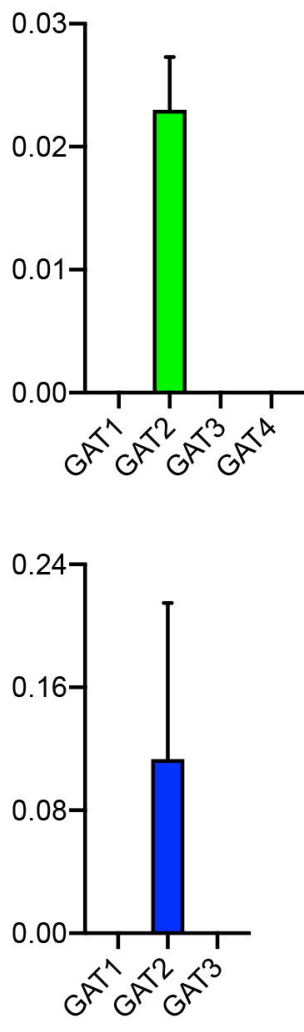

D
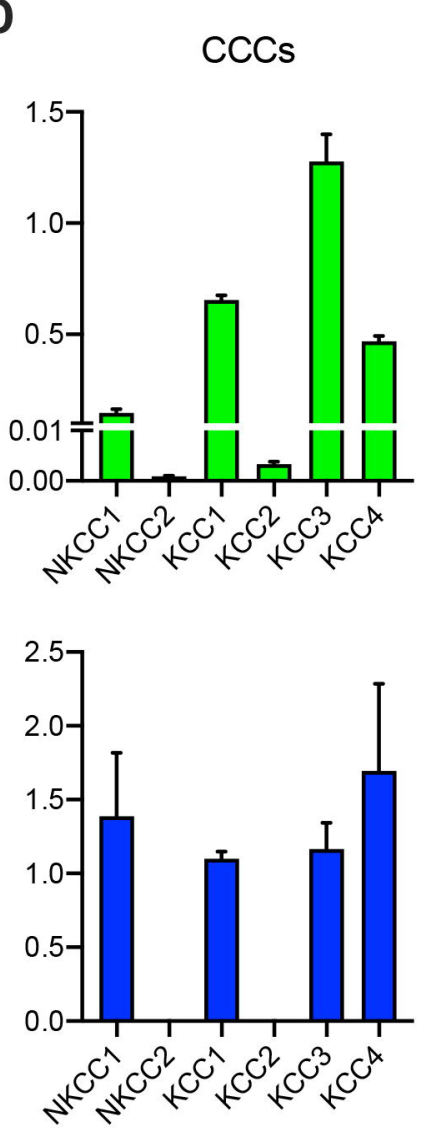
GABA-A receptor subunits

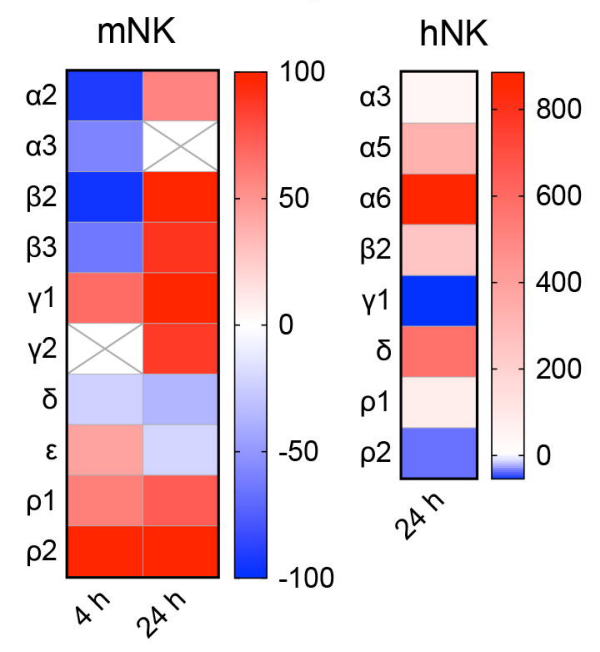

Enzymes

hNK

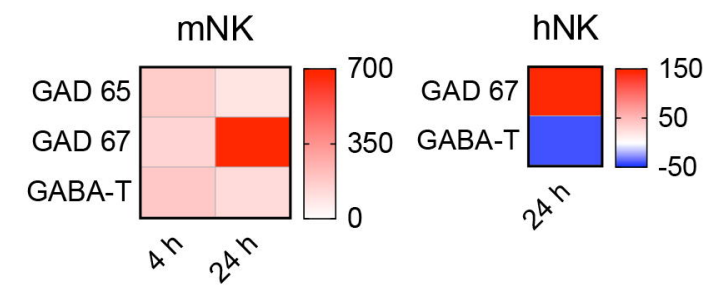

C

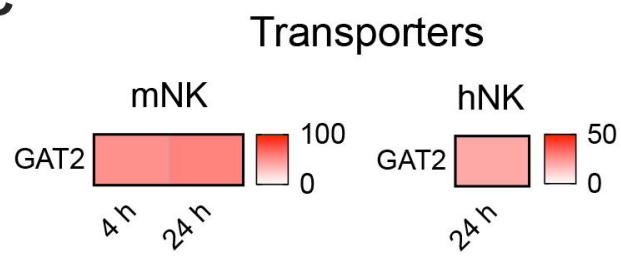

cccs
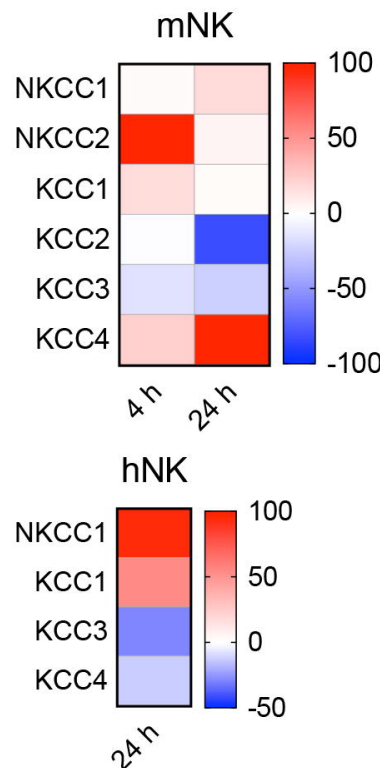
A

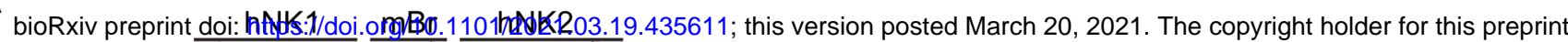
(whichwas not:certified by poer review) is the authq/funder, who has granted bioRxiv a license to display the preprint in perpetuity. It is made T. gondiI availablelunder aCC-BY-NC-ND 4.0 International license.

GAD67

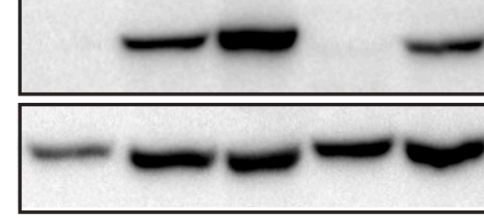

B

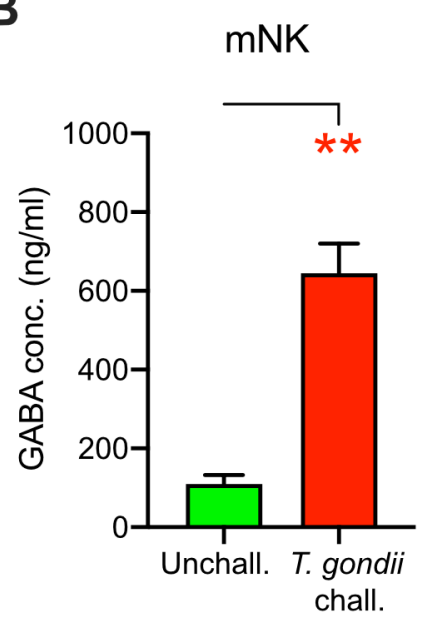

$67 \mathrm{kDa}$

$36 \mathrm{kDa}$

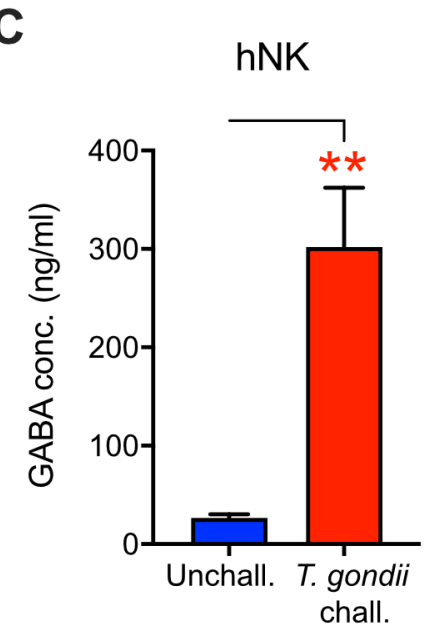

D
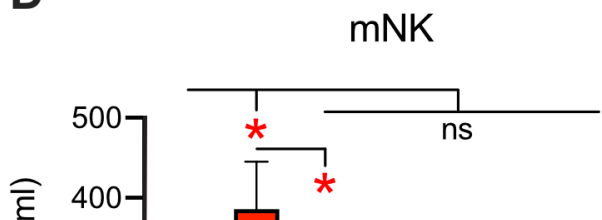

E

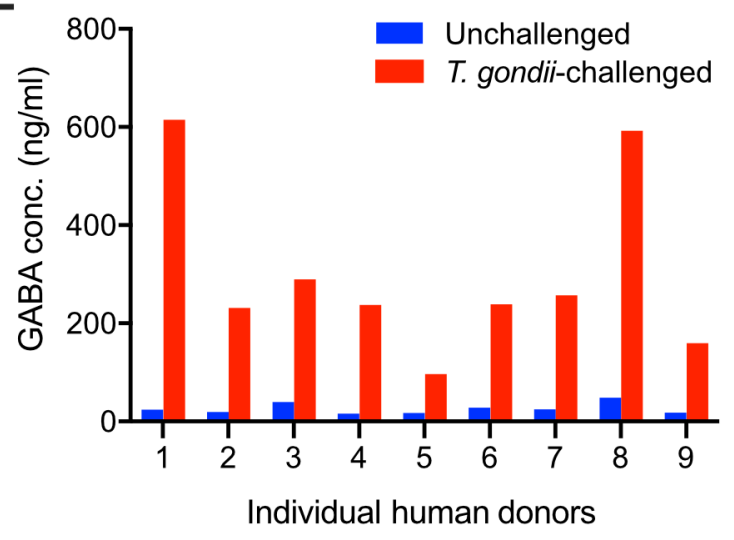

F

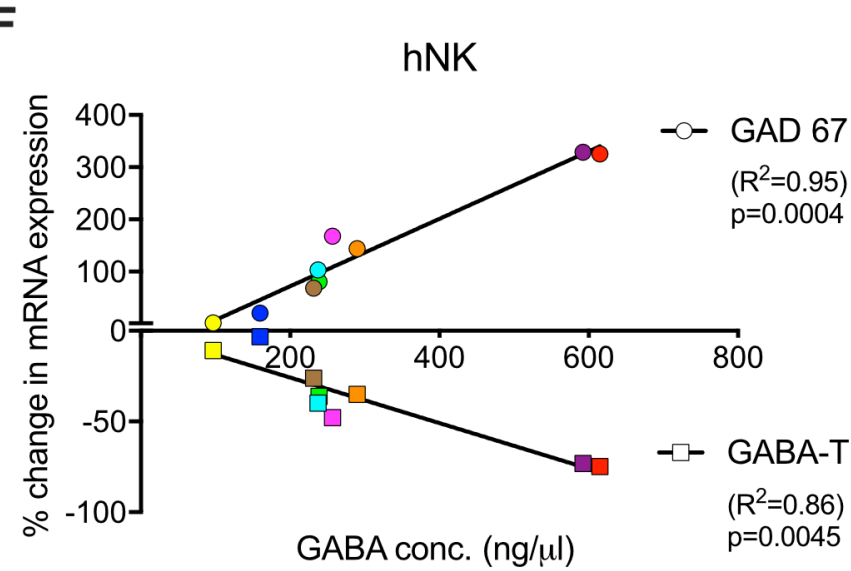

G

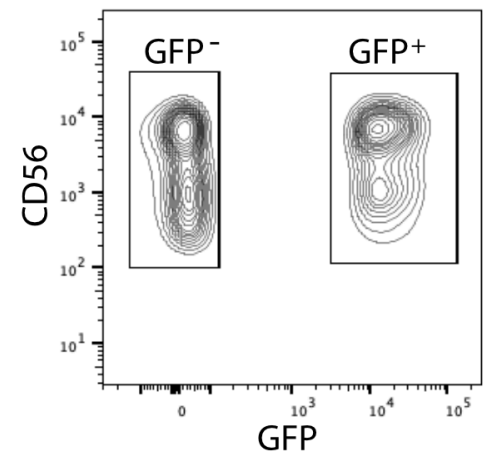

H

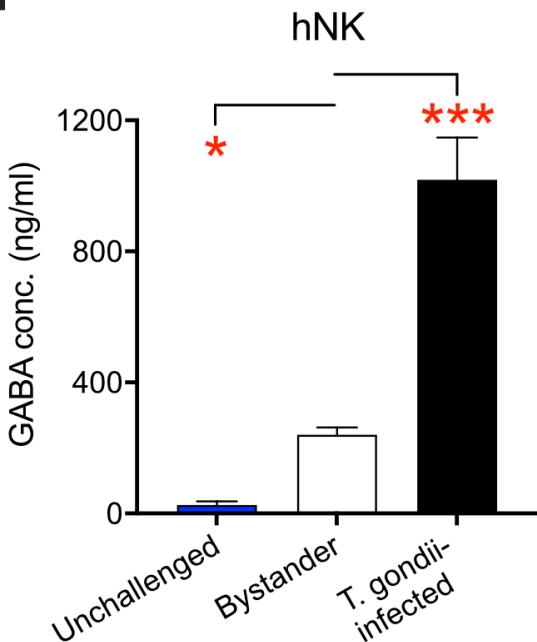

I

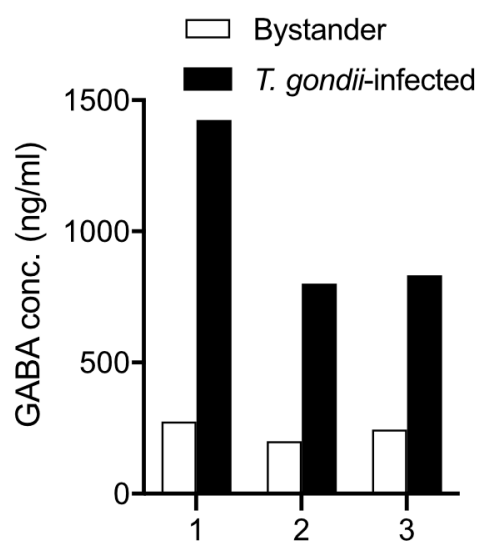

Individual human donors 


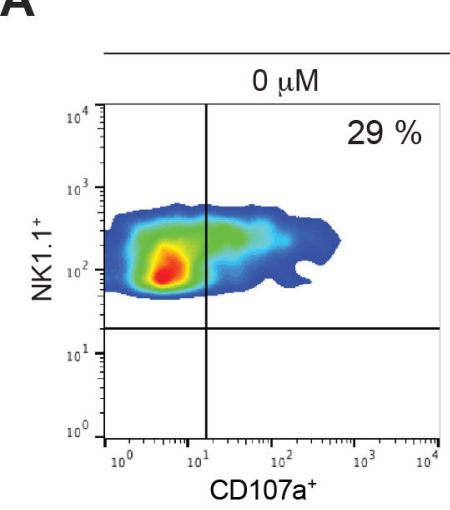

GABA

B

$2 u M$
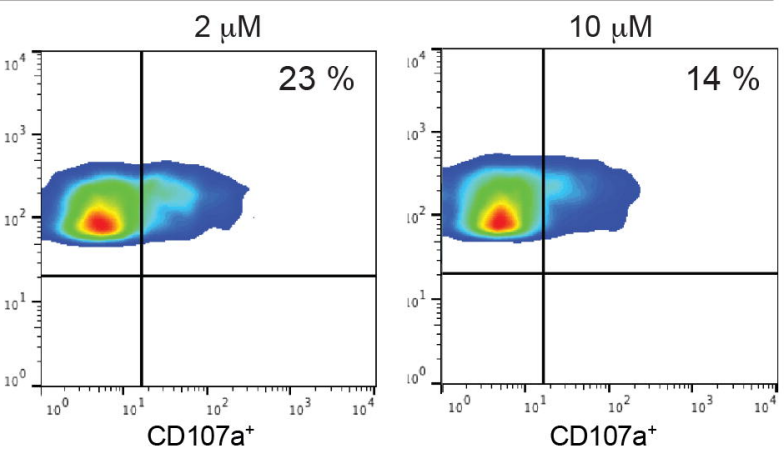

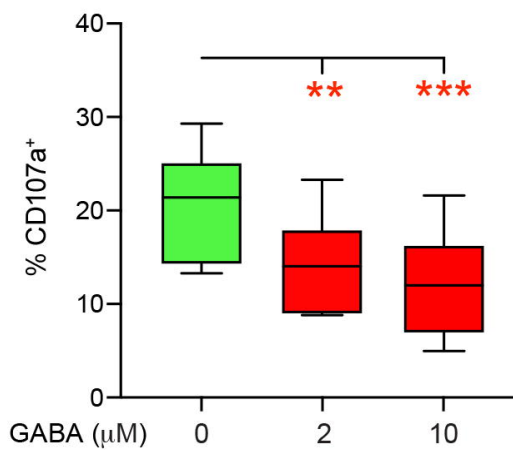

D

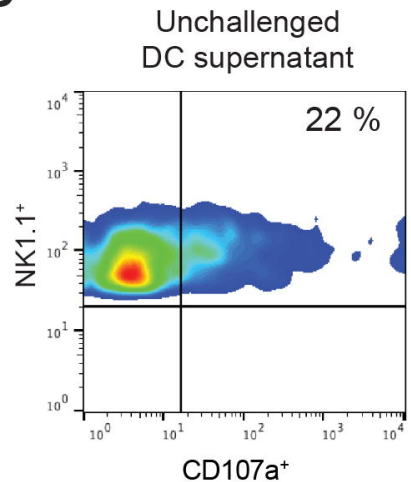

T. gondii-infected DC supernatant

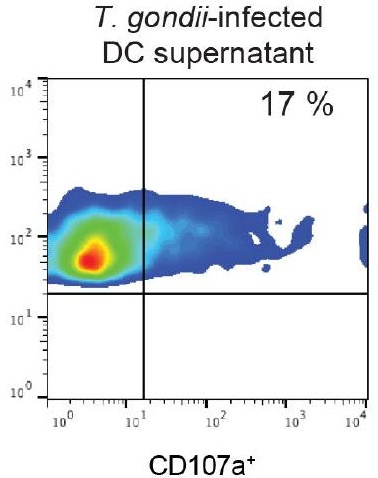

E

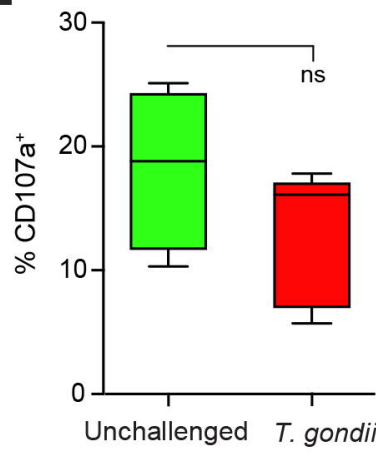

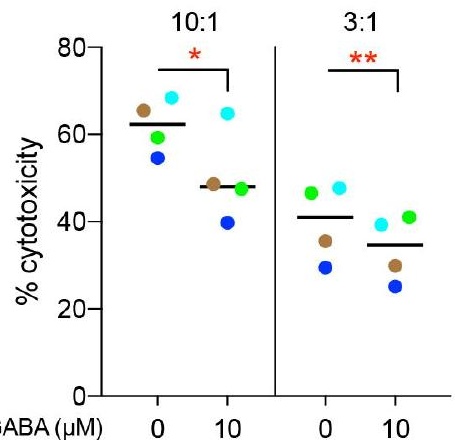



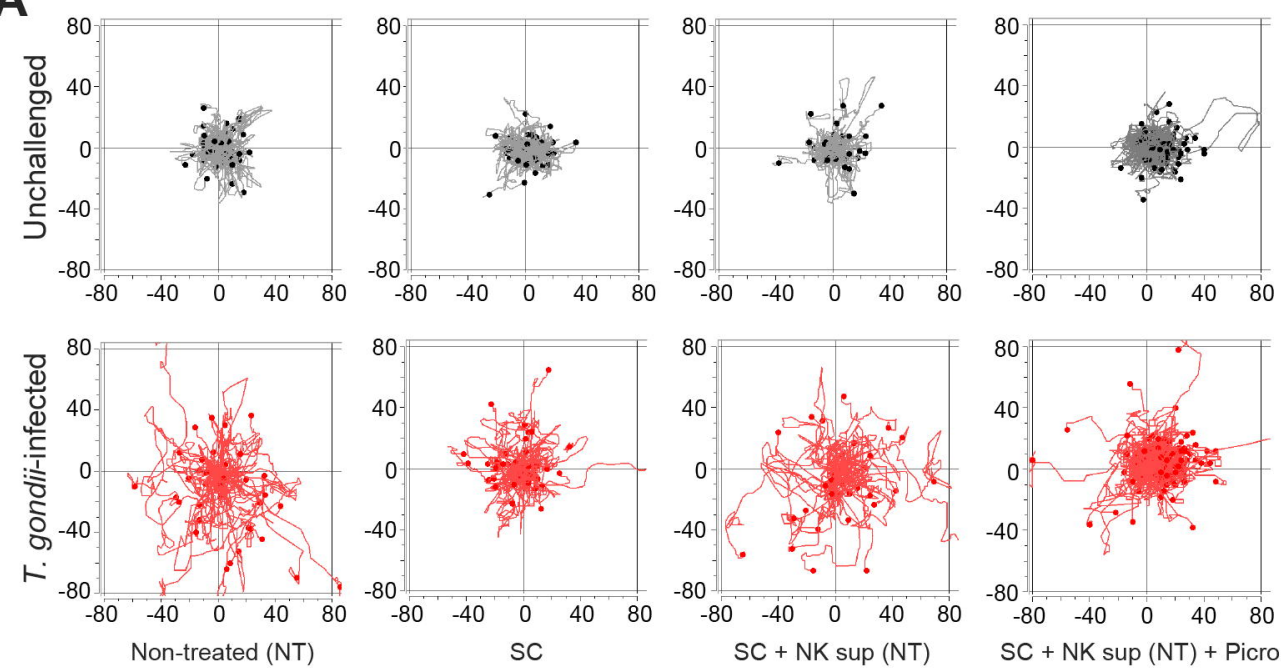

$\square$ Unchallenged $\square$ Infected mBMDC

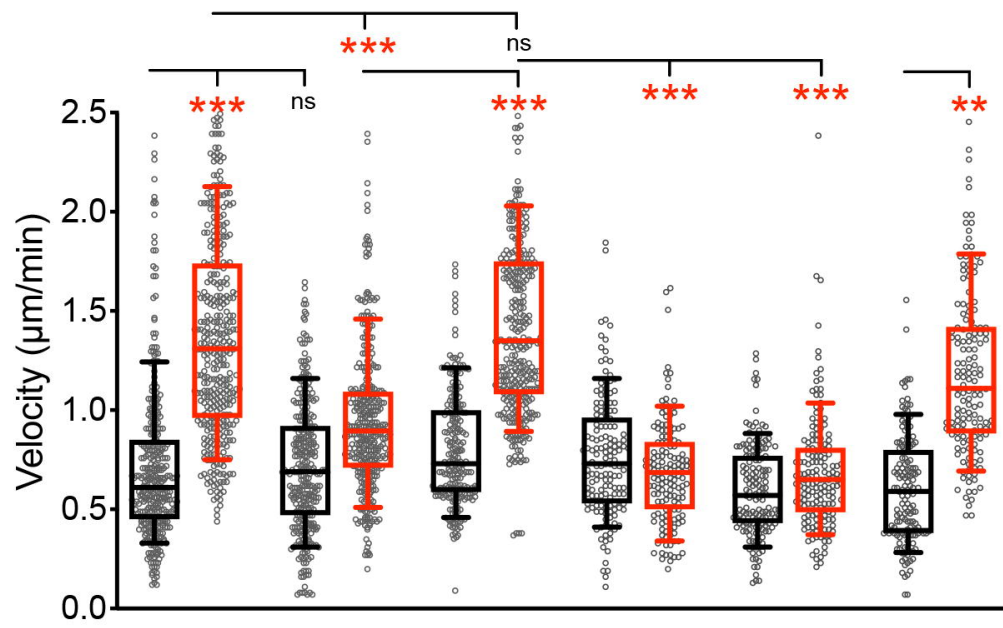

$\mathrm{SC}-\quad-++++++++$

NK sup (NT) - $\quad-\quad-\quad-\quad+\quad+\quad+$

Picrotoxin - $\quad-\quad-\quad-\quad-\quad-\quad+\quad+$

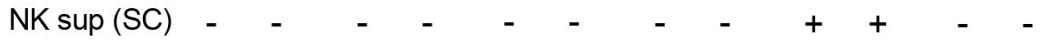

GABA 